\title{
Multiloop calculations with differential equations in $\epsilon$-form.
}

\author{
Roman N. Lee \\ 630090, Budker Institute of Nuclear Physics, Novosibirsk, Russia
}

\begin{abstract}
We review the method of reduction of differential system for multiloop integrals to $\epsilon$-form.
\end{abstract}

\section{Introduction}

New methods of the multiloop perturbative calculations are extremely important in the context of searches for New Physics. On of the most elaborated approaches to the multiloop calculations is the differential equations technique, which was first introduced in Refs. [1-5]. Recent observation made by Henn in Ref. [6] makes this approach yet more powerful. According to this observation, in many cases the differential system for the master integrals can be reduced to the form where the dependence on $\epsilon$ of the right-hand side is restricted to a single factor $\epsilon$. This form (we call it $\epsilon$-form for brevity) allows one to express the coefficients of $\epsilon$ expansion series in terms of Goncharov's polylogarithms. Moreover, the coefficients possess a remarkable property of homogeneous transcendental weight. The advantages of the $\epsilon$-form are even more impressive for the case of several variables. This form gives one a possibility to rewrite differential systems with respect to all variable in one unified 'dlog' form. Since Henn's paper, the differential equations in $\epsilon$-form have been used in many papers, pioneered by Refs.[7, 8].

Finding an appropriate change of functions is not easy. Some partial recipes have been formulated in Refs. $[6,9,10]$. In particular, Ref. [9] assumes linear in $\epsilon$ form (but with nonzero $\epsilon^{0}$ terms) of the right-hand side of the differential system. We stress that, within the approach based on the $\epsilon$-form, the most of the problem's complexity is moved towards achieving this form. Therefore, a general agorithm of finding an appropriate change of functions is very crucial. In Ref. [11] an algorithm of finding the $\epsilon$-form based on rational transformations of the differential system has been presented. In this contribution we briefly review this algorithm.

\section{Reduction to $\epsilon$-form}

Suppose that we have a differential system depending on the parameter $\epsilon$

$$
\partial_{x} \mathbf{J}(x)=\mathbb{M}(\epsilon, x) \mathbf{J}(x),
$$

where the elements of the matrix $\mathbb{M}(\epsilon, x)$ are rational functions of $x$ and $\epsilon$. The systems appearing in the multiloop calculations appear to have only regular singular points, i.e., the the general solution is bounded by $\left|x-x_{0}\right|^{\alpha}$ in the vicinity of any singularity $x_{0}$.

^e-mail: r.n.lee@inp.nsk.su 
The algorithm of Ref. [11] is aimed to find the rational transformation (when it exists)

$$
\mathbf{J}(x) \rightarrow \mathbb{T}(\epsilon, x) \widetilde{\mathbf{J}}(x)
$$

which reduces the system (1) to $\epsilon$-form

$$
\partial_{x} \widetilde{\mathbf{J}}(x)=\epsilon \sum_{k} \frac{\mathbb{M}_{k}}{x-x_{k}} \widetilde{\mathbf{J}}(x),
$$

The algorithm includes three major steps. The first step is to reduce the differential system to the Fuchsian form, i.e., to a form when the elements of $\mathbb{M}$ have only simple poles with respect to $x$. The second step is to normalize the eigenvalues of the matrix residues, i.e., to secure that real parts of all the eigenvalues belong to the interval $[-1 / 2,1 / 2)$. For the systems reducible to $\epsilon$-form this means that all eigenvalues are made proportional to $\epsilon$. Finally, a constant transformation is searched for in order to factor out $\epsilon$, i.e., to reduce the system to $\epsilon$-form.

\subsection{Reducing to Fuchsian form and normalizating eigenvalues.}

Two first steps of the reduction are not specific for the systems depending on parameter and are very natural for generic differential systems. When it concerns the local reduction, the corresponding algorithms are known for a long time. In particular, the possibility to reduce the system to Fuchsian form in the vicinity of a given regular singular point was considered in Ref. [12]. The specific algorithm is given by Barkatou\&Pflügel $[13,14]$. In fact, this algorithm allows one the reduce the system to Fuchsian form in all finite regular singular points. However, the behavior of the system at infinity is not controlled and may become essentially worse ${ }^{1}$.

The Barkatou\&Pfluegel algorithm is based on the transformation generated by the matrix

$$
\mathbb{T}(x)=\overline{\mathbb{P}}+\frac{\mathbb{P}}{x-x_{0}},
$$

where $\mathbb{P}$ is some properly chosen projector. This transformation is singular in one finite point, $x=x_{0}$, and at infinity. One can also generalize this transformation so that it has singularities in any two given points:

$$
\mathbb{T}(x)=\overline{\mathbb{P}}+\frac{x-x_{1}}{x-x_{0}} \mathbb{P} .
$$

In what follows we will call this transformation a balance between $x_{0}$ and $x_{1}$.

Suppose that the expansion of $\mathbb{M}$ at $x=x_{0}$ has the form

$$
\mathbb{M}(x)=\frac{\mathbb{A}_{0}}{\left(x-x_{0}\right)^{p+1}}+\frac{\mathbb{A}_{1}}{\left(x-x_{0}\right)^{p}}+\ldots
$$

with $p>0$. Then, the decisive step of Barkatou\&Pfluegel algorithm can be re-worded as the construction of the projector $\mathbb{P}$ with some prescribed properties. The advance made in Ref. [11] is based on the observation that this step, as well as the algorithm for the normalization of the eigenvalues, imposes requirements only on the image of the projector $\mathbb{P}$, but not on its kernel. Then the idea of the reduction is to apply some constraint also on $\operatorname{ker} \mathbb{P}$ in such a way that the transformation (4) does not increase the pole order at $x=x_{1}$. If the expansion at $x=x_{1}$ has the form $\mathbb{M}(x)=\frac{\mathbb{B}_{0}}{\left(x-x_{1}\right)^{q+1}}+\ldots$, then the constraint is simply that ker $\mathbb{P}$ has to be an invariant space of $\mathbb{B}_{0}$.

\footnotetext{
${ }^{1}$ We remark that it is not always possible to reduce the system to global Fuchsian form even though all its singular points may be regular. This is known due to the Bolibrukh's negative solution [15] of the 21st Hilbert problem. Still, the algorithm of the reduction for the systems where global Fuchsian form is possible is very desirable.
} 


\subsection{Constant transformation}

Let us now assume that it appeared to be possible to secure that the system is Fuchsian and normalized at all singular points. I.e., we have

$$
\partial_{x} \mathbf{J}=\mathbb{M}(\epsilon, x) \mathbf{J}=\sum_{k} \frac{\mathbb{M}_{k}(\epsilon)}{x-x_{k}} \mathbf{J},
$$

and the eigenvalues of all matrices $\mathbb{M}_{k}$ are proportional to $\epsilon$. We need to find an $x$-independent transformation matrix which simultaneously transforms all matrices $\mathbb{M}_{k}(\epsilon)$ to the form $\epsilon \mathbb{S}_{k}$, where $\mathbb{S}_{k}$ are constant matrices. Let $\mathbb{T}(\epsilon)$ be such a matrix. Then we write

$$
\mathbb{T}^{-1}(\epsilon) \frac{\mathbb{M}_{k}(\epsilon)}{\epsilon} \mathbb{T}(\epsilon)=\mathbb{S}_{k}=\mathbb{T}^{-1}(\mu) \frac{\mathbb{M}_{k}(\mu)}{\mu} \mathbb{T}(\mu)
$$

Multiplying this equation by $\mathbb{T}(\epsilon)$ from the left and by $\mathbb{T}^{-1}(\mu)$ from the right, we obtain a linear system

$$
\begin{aligned}
& \frac{\mathbb{M}_{1}(\epsilon)}{\epsilon} \mathbb{T}(\epsilon, \mu)= \mathbb{T}(\epsilon, \mu) \frac{\mathbb{M}_{1}(\mu)}{\mu}, \\
& \vdots \\
& \frac{\mathbb{M}_{m}(\epsilon)}{\epsilon} \mathbb{T}(\epsilon, \mu)=\mathbb{T}(\epsilon, \mu) \frac{\mathbb{M}_{m}(\mu)}{\mu}
\end{aligned}
$$

for the elements of the matrix $\mathbb{T}(\epsilon, \mu)=\mathbb{T}(\epsilon) \mathbb{T}^{-1}(\mu)$. If the general solution of this system (found routinely) determines an invertible matrix, the transformation we are looking for can be chosen as $\mathbb{T}(\epsilon)=\mathbb{T}\left(\epsilon, \mu_{0}\right)$, where $\mu_{0}$ is some arbitrarily chosen number, provided $\mathbb{T}(\epsilon, \mu)$ is nonsingular at $\mu=\mu_{0}$.

One might alternatively use the approach based on 'sampling'. Since we are looking for constant transformation $\mathbb{T}(\epsilon)$ which satisfies

$$
\mathbb{T}^{-1}(\epsilon) \mathbb{M}(\epsilon, x) \mathbb{T}(\epsilon)=\epsilon \mathbb{M}(x),
$$

where $\mathbb{M}(x)$ is the matrix independent of $\epsilon$, we might generate equations for sufficiently many sampling points $a_{1}, \ldots a_{m}$ :

$$
\begin{gathered}
\frac{\mathbb{M}\left(\epsilon, a_{1}\right)}{\epsilon} \mathbb{T}(\epsilon, \mu)=\mathbb{T}(\epsilon, \mu) \frac{\mathbb{M}\left(\mu, a_{1}\right)}{\mu}, \\
\vdots \\
\frac{\mathbb{M}\left(\epsilon, a_{m}\right)}{\epsilon} \mathbb{T}(\epsilon, \mu)=\mathbb{T}(\epsilon, \mu) \frac{\mathbb{M}\left(\mu, a_{m}\right)}{\mu}
\end{gathered}
$$

and use them instead of equations (8). This approach has an advantage due to the possibility to use it in the to the case of differential systems over several variables.

\subsection{What may go wrong?}

- In some, very special, situations it might be not possible to construct the projector with a given kernel due to the condition which should satisfy kernel and image of the projection operator: ker $\mathbb{P} \cap$ $\operatorname{Im} \mathbb{P}=\{0\}$. One might try to achieve global Fuchsian form by introducing the apparent singularities, but then the second step is likely to be not possible due same kind of obstruction. Such obstructions naturally correspond to the systems with regular singularities which can not be reduced to global Fuchsian form (corresponding to the negative solution of 21 st Hilbert problem). 
- The normalization might not result in the proportionality of the eigenvalues to $\epsilon$. This situation might be detected before making the second step. Namely, one has to check that all eigenvalues of the matrix residues have the form $n+k \epsilon$, where $n$ is integer. If this is not the case, the $\epsilon$-form might only be achievable after the variable change. Then, in terms of the original variable, the resulting transformation and system typically involve algebraic functions (square roots).

- Third step may result in degenerate matrix $\mathbb{T}(\epsilon, \mu)$ for all $\mu$ (typically $\mathbb{T}(\epsilon, \mu)=0$ ). If this is so, the $\epsilon$-form of the system is not possible. However, to the best of our knowledge, there is no examples for which this step is not possible to accomplish for any irreducible diagonal block of the matrix $\mathbb{M}$. As to the reduction of the off-diagonal elements, such an obstacle is possible but not very crucial, see next Section.

\section{Using block-triangular form}

The size $n$ of the matrices $\mathbb{M}(\epsilon, x)$ appearing in the differential equations for master integrals may be quite large ( $\sim$ several tens). This may constitute computational complications for the transformations that we need. Fortunately, the very process of the derivation of the differential equations, the IBP reduction, shows that the matrix $\mathbb{M}(\epsilon, x)$ has a block-triangular form with diagonal blocks corresponding to the master integrals of a given sector.

The idea of using this block-triangular form is to apply first the algorithm of the above section to each diagonal block. Suppose that this application results in $\epsilon$-form of all diagonal blocks. Then one may certainly achieve the global reduction to Fuchsian form and normalization of the eigenvalues for the whole matrix, see Section 7 of Ref. [11] for details.

The third step might be not possible to accomplish for the whole matrix. But even if this is the case, one still has almost all benefits of $\epsilon$-form. Indeed, for the column-vector $\mathbf{J}_{1}$ of integrals of a certain sector, one then has the system

$$
\partial_{x} \mathbf{J}_{1}=\epsilon \mathbb{A}(x) \mathbf{J}_{1}+\mathbf{g}(x, \epsilon),
$$

where the inhomogeneous term $\mathbf{g}(x, \epsilon)$ is a linear combination of simpler master integrals, the coefficients being the rational functions of $x$ and $\epsilon$. We assume that these simpler integrals have been already calculated at this point in a sense that we know their expansion in $\epsilon$ at fixed $x$ up to a sufficiently high order as well as their asymptotic behavior at fixed $\epsilon$ and $x \rightarrow x_{k}$. Then we also know the expansion and the asymptotic behavior of $\mathbf{g}(x, \epsilon)$, irrespectively on whether the coefficients of the linear combination are proportional to $\epsilon$. This is the only information that we need to proceed with construction of the solution for $\mathbf{J}_{1}$.

\section{Conclusion}

We have reviewed the algorithm of the reduction of differential system to $\epsilon$-form formulated in Ref.[11]. There is already one published application of this algorithm, see Ref. [16], with several more applications coming in the nearest future.

The algorithm formally applies to the differential systems with respect to one variable. However, it appears that it is also extremely helpful for the multivariate case, although it requires more heuristic work.

\section{Acknoledgments}

I am grateful to A.A. Pomeransky for valuable discussions. This work is supported by RFBR grant No. 15-02-07893. 


\section{References}

[1] A.V. Kotikov, Phys. Lett. B254, 158 (1991)

[2] A.V. Kotikov, Phys. Lett. B259, 314 (1991)

[3] A.V. Kotikov, Phys. Lett. B267, 123 (1991)

[4] E. Remiddi, Nuovo Cim. A110, 1435 (1997), hep-th/9711188

[5] T. Gehrmann, E. Remiddi, Nucl. Phys. B 580, 485 (2000), hep-ph/9912329

[6] J.M. Henn, Phys.Rev.Lett. 110, 251601 (2013), 1304.1806

[7] J.M. Henn, V.A. Smirnov, JHEP 1311, 041 (2013), 1307.4083

[8] J.M. Henn, A.V. Smirnov, V.A. Smirnov, JHEP 1307, 128 (2013), 1306. 2799

[9] M. Argeri, S. Di Vita, P. Mastrolia, E. Mirabella, J. Schlenk et al., JHEP 1403, 082 (2014), 1401.2979

[10] T. Gehrmann, A. von Manteuffel, L. Tancredi, E. Weihs, JHEP 1406, 032 (2014), 1404.4853

[11] R.N. Lee, J. High Energy Phys. 1504, 108 (2015), 1411.0911

[12] J. Moser, Mathematische Zeitschrift 72, 379 (1960)

[13] M.A. Barkatou, E. Pflügel, Computing super-irreducible forms of systems of linear differential equations via Moser-reduction: a new approach, in Proceedings of the 2007 international symposium on Symbolic and algebraic computation (ACM, 2007), pp. 1-8

[14] M.A. Barkatou, E. Pflügel, Journal of Symbolic Computation 44, 1017 (2009)

[15] A.A. Bolibrukh, Proc. Steklov Inst. Math. 206, 145 (1995)

[16] R.N. Lee, K.T. Mingulov, Phys. Lett. B 757, 207 (2016), 1602.02463 